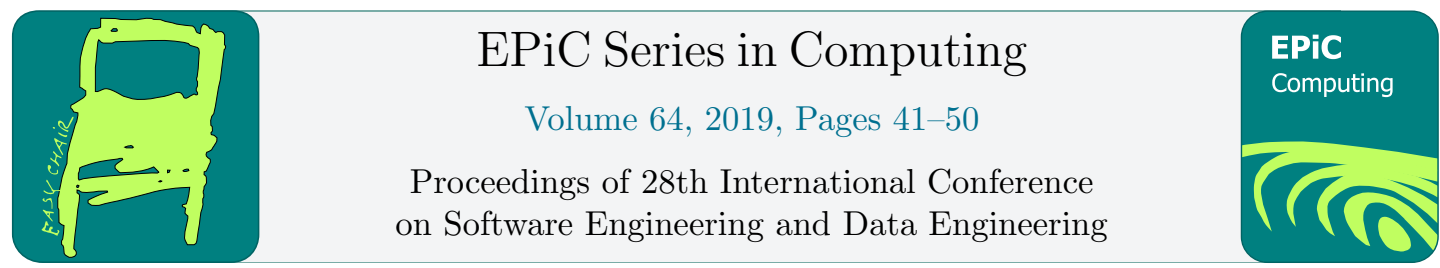

\title{
Scalable Correlated Sampling for Join Query Estimations on Big Data
}

\author{
David S. Wilson ${ }^{1}$, Wen-Chi Hou ${ }^{2}$, and Feng $\mathrm{Yu}^{1}$ \\ 1 Youngstown State University, Youngstown, OH, USA \\ dswilson@student . ysu .edu, fyu@ysu.edu \\ 2 Southern Illinois Univeristy, Carbondale, IL, USA \\ hou@cs.siu.edu
}

\begin{abstract}
Estimate query results within limited time constraints is a challenging problem in the research of big data management. Query estimation based on simple random samples performs well for simple selection queries; however, return results with extremely high relative errors for complex join queries. Existing methods only work well with foreign key joins, and the sample size can grow dramatically as the dataset gets larger. This research implements a scalable sampling scheme in a big data environment, namely correlated sampling in map-reduce, that can speed up search query length results, give precise join query estimations, and minimize storage costs when presented with big data. Extensive experiments with large TPC-H datasets in Apache Hive show that our sampling method produces fast and accurate query estimations on big data.
\end{abstract}

\section{Introduction}

Big data is everywhere. Approximately 2.5 quintillion bytes (2.5 billion Gigabytes) of data is produced each day. Ninety percent of the data created in the world has been created in the past two years [9]. To put that into perspective, IBM created the IBM Model 350 Disk File in 1956. It was the size of a compact-size car, and had a storage capacity of five megabytes. If one were to place these machines side by side, based off the amount of data we use in one day, they would circle the earth nine thousand one hundred and ninety times. With the sizes of company databases reaching terabytes and even petabytes, and at the speed of which this data is being accumulated, the need for query optimization has never been so high.

Query optimization [8] is the process of using statistics about the database, as well as assumptions about the attribute values, to acquire the best execution plans for queries. Some databases are large, and data streams in so fast that queries can take minutes, hours, even days to process. Correlated sampling [16] is a statistical summary scheme for a database, and through unique methods, aims to provide a fast and precise result size estimation for queries with joins and arbitrary selections. The aim of this work is to extend the methods of CS2, apply them to join query estimations on big data, and present the findings. 
The rest of this work is organized as follows. Section 2 states the background of the problem. Correlated sampling on big data is elaborated in section 3. Experiment results are presented in section 4 . Section 5 concludes the work.

\section{Background}

\subsection{Big Data Management}

Big Data may just be one of the most misunderstood terms in the technology field. It is miscommonly referred to as a large volume of data. While not entirely incorrect, there is much more to Big Data then just size. In the following sections, the origins of big data, as well as what defines data as "Big data", will be discussed.

The term "Big Data" was first coined in 1998 by John Mashey of Silicon Graphics, Inc., although this is debated [13]. Others had written about big data before this date, but Mr.Mashey was the first that used the term in the context of computing. Even though the term Big Data was created in the 90's it was not until the early 2000's that it took the form of what is is considered today. In February 2001, Doug Laney created the three V's of Big Data, which are Volume, Variety, and Velocity. [6]

The Apache Hadoop framework [15] consists of multiple modules, each having its own distinctive responsibilities. Hadoop Common is the storehouse for other Hadoop Modules. It holds all of the files in which the other Hadoop Modules need to run properly. Hadoop Distributed File System, or HDFS for short,[2] deals with the storage of data of a Hadoop cluster. A major issue with storing streaming, large sets of data, is hardware failure. HDFS is built to combat this, by using a process called replication. HDFS consists of a name node, which stores all meta data of all files stored. It also has data nodes as well, which hold all of the actual data. Each data node consists of a multitude of blocks, with each block of data being stored into 3 different data node locations in the cluster. If at any time there is a node that fails, or a machine in the cluster fails, another block copy is made on another node or machine.

\subsection{Database Systems on Big Data}

With the rise of big data, many database systems have been developed on big data for scalable data management and processing such as Hive[12], HBase[14], Dynamo[5], etc. Among them, Hive was created to make it easier for users to be able to use Hadoop's Map-Reduce and HDFS without an advanced knowledge of Java. As mentioned earlier, Hive uses a similar language to SQL, called HQL or Hive Query Language. With the use of this language users are able to perform data queries, as well as summarize and analyze data. Users can use traditional command line to work in Hive, or us HWI (Hive Web Interface). HWI is a graphical user interface, or GUI, that simplifies the use of hive.

\subsection{Join Graph of a Database}

Definition 1. (Join Graph) A join graph [11] is a visual representation of a database in which the flow of joins is explained. It can be created to take into consideration the relational type of joins,(many-to-many, many-to-one, one-to-one), and also if there are multiple attributes that can be used within the join. It is a general representation in which the join relations of a database are mapped out [16]. 


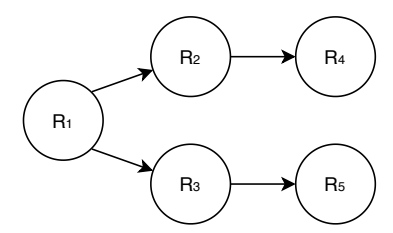

Figure 1: A Basic Join Graph

Definition 2. (Joinable Relations) Two relations considered joinable, $R_{i}$ and $R_{k}, i \neq k$, when there is a path with length $\geq 1$ between the relations $R_{i}$ and $R_{k}$ [16].

Definition 3. (Joinable Tuples) Under the assumption that $R_{i}$ and $R_{k}$ is a joinable relation, a tuple in $R_{i}$,denoted by $t_{i}$, and a tuple in $R_{k}$, denoted $t_{k}$, is considered joinable if $t_{i}$ can find a match $t_{i+1}$ in $R_{i+1}, t_{i+1}$ can find a match $t_{i+2}$ in $R_{i+2}$, and $t_{k-1}$ can find a match $t_{k}$ in $R_{k}$ [16].

Figure 1 is a basic join graph of a database. It shows that Relation 1 denoted as $R_{1}$, has joinable attributes with Relation 2 , as well as Relation 3, denoted with $R_{2}$ and $R_{3}$ respectively. $R_{2}$ has joinable attributes with Relation 4 , denoted with $R_{4}$, but does not have any joinable attributes with $R_{3}$ or Relation 5 , denoted with $R_{5}$. $R_{3}$ has joinable attributes with $R_{5}$, but no joinable attributes with $R_{2}$ or $R_{4}$.

\subsection{Random Sampling}

Random sampling has been widely adopted for query size estimations. Simple Random Sample Without Replacement, or SRSWOR, $[7,10]$ has previously been tested as a random sample synopsis. A SRSWOR of each relation is taken separately, and then the resulting independent samples are joined. Unfortunately, the final results end in massive errors of the join size estimation [3]. SRSWOR is beneficial if one is only seeking to get a size estimation on an individual relation.

\subsection{Join Synopses}

While Join Synopses (or JS) [1] use SRSWOR in its mechanics, the process does adds a join correlation between individual relations, causing a much better relative error. JS uses foreign key joins and computes samples of a small set of joins, procuring samples of all possible joins in a schema. These samples are then stored, and joined with individual SRSWOR relations to form a unbiased finalized set of correlated random tuples that can be used for query estimation. A major draw back this approach is it is quite time consuming in the sampling process. JS requires a SRSWOR on each relation in the database followed by correlated sampling on each joinable relation along the path in the join graph. For a path in a join graph consists of $n$ relations starting from $R_{1}$ to $R_{n}, O\left(n^{2}\right)$ sampling operations have to be performed to generate a JS. Figure 2a depicts an example of JS creation with three joinable relations in a path of the join graph. 


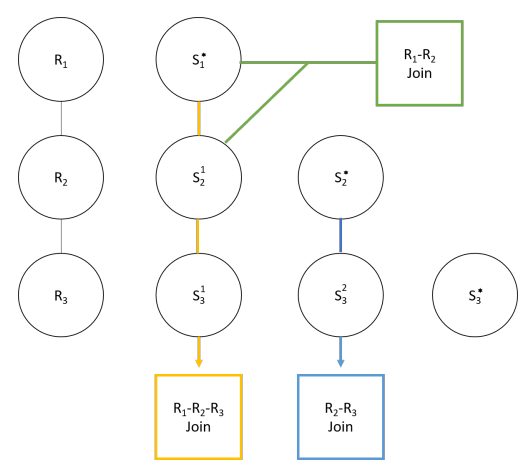

(a) Sampling of Join Synopses

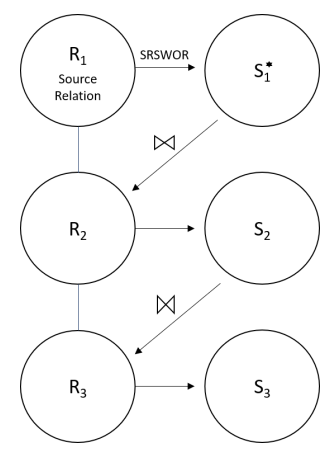

(b) Correlated Sampling of CS2

Figure 2: Join Synopses and Correlated Sampling

\section{Correlated Sampling on Big Data}

\subsection{Correlated Sample Synopsis}

To mitigate the sampling costs of JS, Yu et al. proposed Correlated Sample Synopsis (or CS2) [16] which is a statistical summary for a database and can be used for both query estimation and approximate query processing (AQP). The purpose of CS2 is to create a unbiased, fast, and precise estimation for queries with all types of joins and selections. CS2 preserves join relationships between tuples and their relations. Unlike JS, CS2 doesn't require SRSWOR on every relation in the join graph but employs a special value called Join Ratio (or JR) with a Reverse Estimator (or RV Estimator) to provide unbiased join query estimations.

Figure $2 \mathrm{~b}$ illustrates a sample example of the process that transpires once the source relation and path selection are decided on. A simple random sample without replacement is performed on the source relation denoted as $R_{1}$, with results of this SRSWOR being placed in a sample relation, denoted as $S_{1}^{*}$ with a star to signify it is a SRSWOR of $R_{1}$. The next relation, denoted $R_{2}$ is now ready to be moved to. To create the correlation between relations and preserve the join relationships, $S_{1}^{*}$ is joined with $R_{2}$, with the results being placed into a second sample relation, denoted as $S_{2}$. In this example, the source relation only consists of one edge to another relation. In the case that there are multiple edges to multiple relations, one would exhaust all possibilities by creating sample relations for each relation until all edges are accounted for. Relation three, denoted as $R_{3}$, is then joined with $S_{2}$ with the results being placed in the third sample relation, denoted as $S_{3}$. The combination of all of the sample relations is considered the CS2 synopsis.

\subsection{Sampling in Map-Reduce}

In big data file systems, such as HDFS, access to data is required to be translated into operations of map and reduce. Sampling operations on traditionally centralized database systems are not exceptional when converted to the environment of map-reduce. JS and CS2 preserves the joinable relations of tuples between sampled relations by performing join operations which are categorized into two different operations in big data, namely map join and reduce join. Given two relation tables $R$ and $S$, when joining $R$ and $S$, denoted by $R \bowtie S$ in traditional databases, if $R$ is smaller than $S$ and can be fit into the memory heaps of data nodes in a big data cluster, then $R$ is mapped to all data nodes where $S$ is distributed where a map join is performed, 


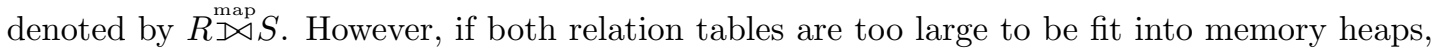
then a common map-reduce procedure will be initiated to compute the join result, called reduce

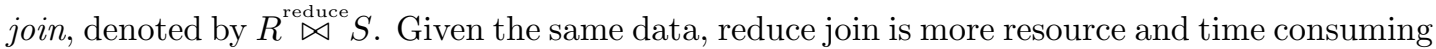
compared to map join. To mitigate the sampling cost, correlated sampling on big data aims to control the sample size small enough and use map join as much as possible during the process.

\subsection{Join Graph Path Creation and Source Relation Selection}

Correlated Sampling begins with the creation of a join graph of the database, as well as the determined size preferred for the sample relations. A source relation selection must then be made.

It is important to note, CS2 does work with any join relationship (one-to-many, many-toone, many-to-many). However, when selecting your sampling path and source relation it is suggested to use and follow a many-to-one relationship, as using a one-to-many or many-tomany relationship can cause the synopsis to grow considerably, subtracting from the overall number of sample tuples that can be taken from the source relation. For a complicated join graph, multiple source relations are allowed to follow many-to-one-relationships.

\subsection{Correlated Sampling in Map-Reduce}

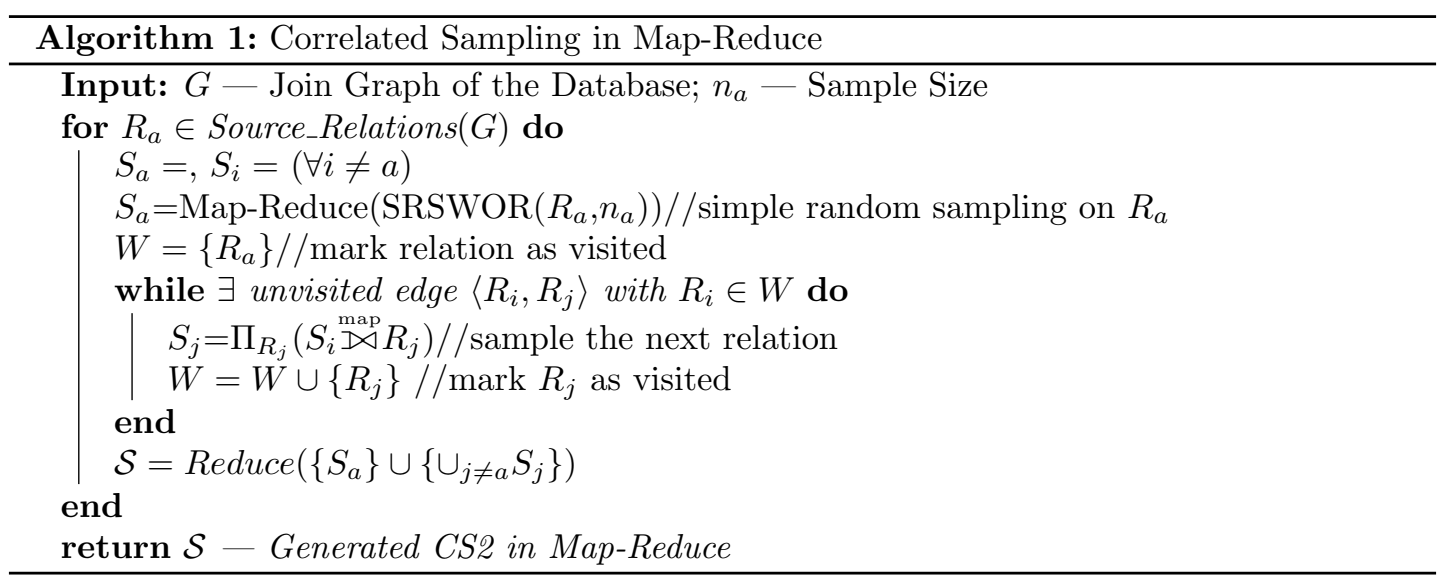

Algorithm 1 shows the process of correlated sampling in map-reduce. For a complicated join graph, multiple source relations are allowed and the join graph can be partitioned into multiple join graph paths. For each source relation $R_{a}$ in a join graph path, a SRSWOR is first performed by a map function with a small number of $n_{a}$ tuples sampled from $R_{a}$. To retain the joinable relations, the correlated tuples are collected in $R_{j}$ when map joined with $S_{i}$, which is the parent joinable relation along the join path. Note that, the join graph path in CS2 are recommended to follow many-to-one relationships; therefore, the size of $S_{j}$ is no larger than $S_{i}$ and the map join can be continued along the join path since the sample size will generally decrease. Finally, a reduce function is initiated to collect all sampled relations into $\mathcal{S}$ as the generated CS2 in map-reduce. Section 4 includes the details of implementing correlated sampling in Apache Hive. 


\subsection{Query Estimation}

The process of query estimation is taking the results from a sample query, and using said results to estimate query result sizes.

\subsubsection{Source Query Estimation}

Source Query Estimation, is the process of estimating query results using sample queries that includes the source relation. Referring back to Figure $2 \mathrm{~b}$, a source query would be considered a join of relations $S_{1}^{*}$ and $S_{2}$, or a join between relations $S_{1}^{*}$ and $S_{3}$. The results of these joins could then be used to estimate the join query size of joins between $R_{1}$, and $R_{2}$, as well as $R_{1}$ and $R_{3}$.

Given $S_{1}^{*}$, a SRSWOR of the source relation $R_{1}$ the estimation of the query result is estimated by

$$
\widehat{Y}_{\text {source }}=\frac{N_{1}}{n_{1}} \sum_{i=1}^{n_{1}} y_{i}
$$

where $N_{1}=\left|R_{1}\right|$ and $n_{1}=\left|S_{1}\right|$, and $y_{i}$ is the number of result tuples generated by the $i$ th tuple in $S_{1}^{*}$.

\subsubsection{No-Source Query Estimation}

No-Source Query Estimation, is the process of estimating query results using sample queries that do not include the source relation. In Figure 2b, a join of $S_{2}$ and $S_{3}$ would be considered a No-Source Query. Due to the conditions of a No-Source query not containing a SRSWOR based off the source relation, additional steps must be taken for accurate estimation. Joinable Tuple Sampled Ratio, or JR, is a procedure of backtracking to the source relation in a no-source query (reverse sampling), and supplying it with the ability to estimate the join query size.

Given $R_{h}$ the top relation in the given query and $S_{h}$ the correlated sample of $R_{h}$, the estimation of the query result is estimated by

$$
\widehat{Y}_{\text {no_source }}=\frac{N_{1}}{n_{1}} \sum_{j=1}^{n_{h}} r_{j} y_{j}
$$

where $n_{h}=\left|S_{h}\right|, y_{j}$ is the number of result tuples generated by the $j$ th tuple in $S_{h}$, and $r_{j}$ is the JR value associated with the $j$ th tuple in $S_{h}$. The JR value of $r_{j}$ associated with a tuple $t_{i}$ in $R_{h}$ equals the total number of joinable tuples in $S_{1}$ divided by the total number of joinable tuples in $R_{1}$ [16].

\section{Experiments}

\subsection{Experiment Setup}

A cluster of five nodes on a remote server were created in the Sarah Cloud created in YSU Data $\mathrm{Lab}^{1}$. This cluster consists of two master nodes and three worker nodes. Master Node One has four Intel Xeon CPU's (E5-2630 v4 @ 2.20 GHz) and 16GB of RAM. Master Node Two has two Intel Xeon CPU's (E5-2630 v4 @ 2.20 GHz) and 10GB of RAM. All worker nodes consist

\footnotetext{
${ }^{1}$ http://datalab.ysu.edu
} 


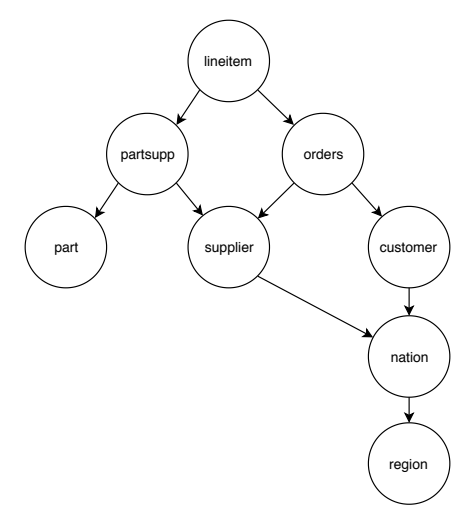

Figure 3: Sampling Graph of the TPC-H Dataset

of the same setup, a Intel Xeon CPU (E5-2630 v4 @ 2.20 GHz) processor and 8GB of RAM. The cluster is running Hadoop with Hive setup.

Two datasets are used, both datasets are generated using TPC-H benchmark[4]. The first dataset created has a total size of 1GB. The second dataset created has a total size of 10GB. Each dataset holds eight relations. The relations are Lineitem, Customer, Orders, Partsupp, Part, Supplier, Nation, and Region.

The following steps are taken to prepare for experimentation on the big data.

Step 1. Using the source dataset, a source relation as well as a join graph path must be decided on. Lineitem table holds the most many-to-one relationships, and is selected as the source relations. The sampling path that was chosen based on relationships was as follows:

Lineitem $\rightarrow$ Orders, Lineitem $\rightarrow$ Partsupp, Orders $\rightarrow$ Customer, Partsupp $\rightarrow$ Part, Partsupp $\rightarrow$ Supplier, Customer $\rightarrow$ Nation, Nation $\rightarrow$ Region

Step 2. A empty set must be created to store the samples of the source dataset. The 1GB and 10GB datasets were denoted as tpch1g and tpch10g respectively. The sample datasets were denoted as s_tpch1g and s_tpch10g respectively.

Step 3. Before creating the SRSWOR a sample dataset size must be selected. The decision was made that the sample dataset size would be one percent of the source dataset. The HQL to create the SRSWOR is as follows:

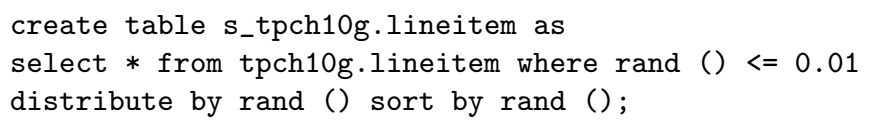

The HQL lines distribute by rand (), and sort by rand () were added to create a higher rate of randomness. distribute by rand ( ) takes the entire set of tuples from a table, and distributes them randomly to different reducers. sort by rand () then takes these sets of random tuples and sorts them randomly on each reducer.

Step 4. Using the created SRSWOR, and following the join graph path, the rest of the sample relations are constructed.

\section{$4.2 \quad$ Results}

Overall, a total of 15 queries were tested five times each, over both the 1GB and 10GB source dataset, as well as the 1GB and 10GB sample dataset. The original dataset, is denoted as the 
Table 1: Query Search Times

\begin{tabular}{|l|l|l|l|l|l|}
\hline \multirow{2}{*}{ Dataset } & Type & Average (sec) & $\begin{array}{l}\text { Average } \\
\text { Speedup (1.0x) }\end{array}$ & High (sec) & Low (sec) \\
\hline \multirow{2}{*}{ 1GB } & source & 16.26 & \multirow{2}{*}{3.05} & 23.24 & 11.57 \\
\cline { 2 - 4 } & sample & 5.33 & & 7.81 & 3.71 \\
\hline \multirow{2}{*}{$10 \mathrm{~GB}$} & source & 437.49 & \multirow{2}{*}{45.91} & 1692.87 & 69.57 \\
\cline { 2 - 3 } \cline { 5 - 6 } & sample & 9.53 & 14.84 & 7 \\
\hline
\end{tabular}

"source" on all graphs, and the correlated sample dataset, is denoted as "sample". Discussion about the results for the 1GB dataset will be presented first, followed by the 10GB dataset results, and finishing with discoveries made through the testing phase. The datasets were tested on speed of queries, as well as accuracy of the estimations.

For accuracy tests, we compare the estimated query results by CS2 ( $\left.Q_{\text {estimated }}\right)$ with the ground truth query results from the source database $\left(Q_{\text {ground_truth }}\right)$ and calculate the absolute relative error. The formula of the absolute relative error is given by:

$$
\text { Absolute Relative Error }=\left|\frac{Q_{\text {ground_truth }}-Q_{\text {estimated }}}{Q_{\text {ground_truth }}}\right| \times 100 \%
$$

1GB Experiments. Depicted in Figure 4, the 1GB dataset source dataset tests averaged a total query time of 16.26 seconds, with a high average of 23.24 seconds in Query 11, and a low average of 11.57 seconds in Query 10. The 1GB sample dataset tests averaged 5.33 seconds, with a high average of 7.81 on Query 12, and a low average of 3.71 on Query 14. The average speed up from the sample, over the source would be a $205 \%$. The largest speed up was Query 14 at $321.71 \%$, and the lowest speed up was Query 9 at $141.65 \%$. The results were impressive on the $1 \mathrm{~GB}$ dataset with the sample dataset processing much faster than the source.

The average count of tuples for the source dataset was 2,520,952. The average count of tuples for the sample dataset was 25,385. The average join estimation results based off source join estimation was 2,538,533. The average Relative Error for the 1GB dataset was .96\%. The highest relative error was $2.50 \%$ on Query 3 with the source dataset holding 592,794 tuples, the sample dataset holding 6076 tuples and source join estimation showing 607,600 tuples. The lowest relative error was $.09 \%$ on query 7 , with the source dataset showing 24,877 , the sample dataset showing 249, and the source join estimation showing 24,900.

10GB Experiments. Depicted in Figure 5, the 10GB dataset source dataset tests averaged a total query time of 437.49 seconds, with a high average of 1692.87 seconds in Query 11, and a low average of 69.57 seconds in Query 7. The $1 \mathrm{~GB}$ sample dataset tests averaged 9.53 seconds, with a high average of 14.84 on Query 11, and a low average of 7 on Query 3. The average speed up from the sample, over the source would be a $4489.44 \%$. The largest speed up was Query 11 at $16,071.90 \%$, and the lowest speed up was Query 2 at $758.39 \%$. This shows that the larger the dataset, the better CS2 performs in speed.

The average count of tuples for the source dataset was 25,208,072. The average count of tuples for the sample dataset was 251,168 . The average join estimation results based off source join estimation was $25,116,820$. The average Relative Error for the 10GB dataset was $.34 \%$. The highest relative error was $.76 \%$ on Query 7 with the source dataset holding 248.493 tuples, the sample dataset holding 2466 tuples and source join estimation showing 246600 tuples. The lowest relative error was $.01 \%$ on query 15 , with the source dataset showing $6,047,718$, the sample dataset showing 60474, and the source join estimation showing 6,047,400. The results show that not only does CS2 speed up the larger the data gets, but its accuracy also improves. 


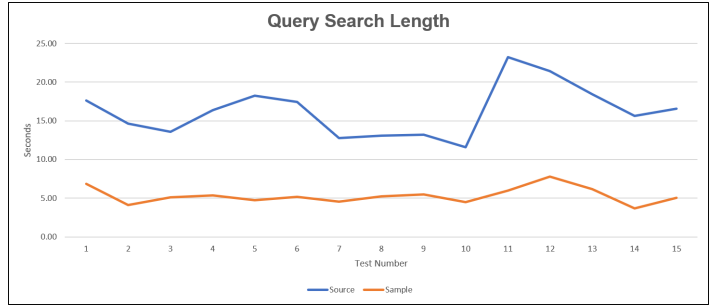

(a) 1GB Dataset Query Search Length Results (Seconds)

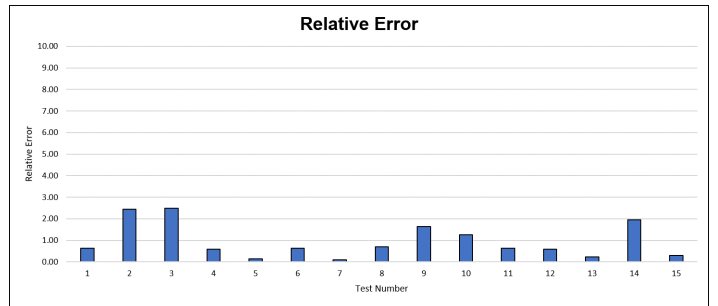

(b) 1GB Dataset Relative Error Results (\%)

Figure 4: Experiments of 1GB Dataset

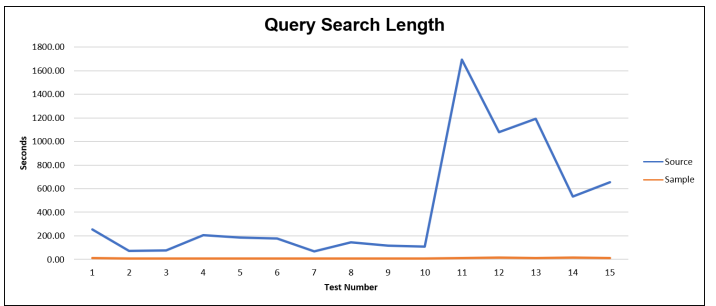

(a) 10GB Dataset Query Search Length (Seconds)

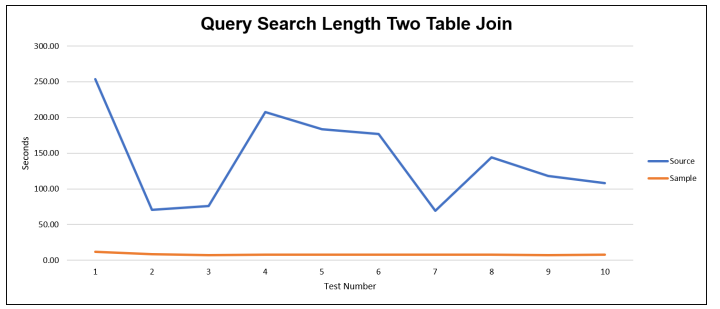

(c) 10GB Dataset Two Relation Join Query Results (Seconds)

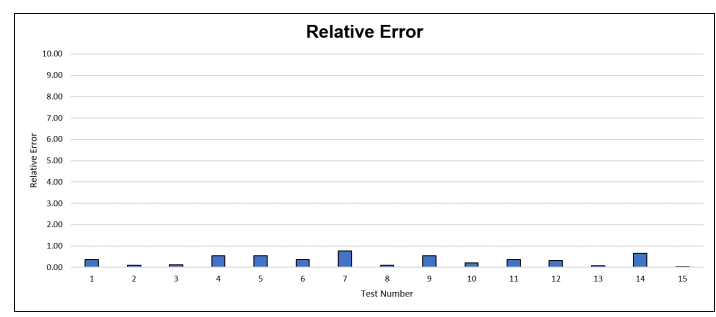

(b) 10 GB Dataset Relative Error Results (\%)

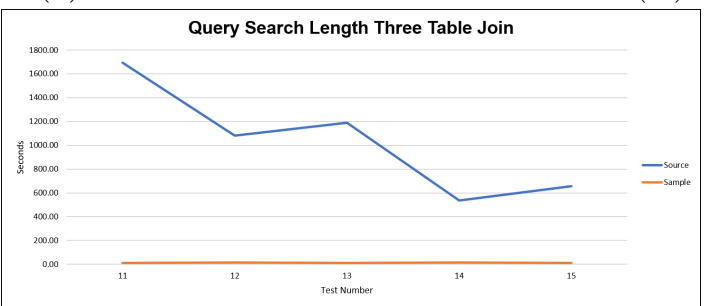

(d) 10GB Dataset Three Relation Join Query Results (Seconds)

Figure 5: Experiments on 10GB Dataset

The advantage of CS2 in map-reduce was recognized. Not only does CS2 speed up the join queries, but when moving from a two relation join, to a three relation join, the time increase is very minimal for CS2. In Figure 5c, the two relation join query results, CS2 holds at about a seven second average while the source averages around 180 seconds. When the queries switched to a three relation join in Figure 5d, the average for CS2 bumps up to about 10 seconds, while the source relation explodes and averages about 1100 seconds per query.

\section{Conclusion and Future Works}

In this research, the use of correlated sampling on big data was introduced. It was discovered that not only does CS2 in map-reduce maintain the accuracy of tuples from its samples in join query estimation, but increases in precision as the dataset grows larger. CS2 in map-reduce also maintained a constant speed and did not increase much as the datasets expanded in size. When the source relation query search length ballooned in size with the three relation joins, CS2 continued do produce low search query lengths. Based off the results, CS2 in map-reduce proved 
to be successful in query optimization and more efficient in regards to scalability and accuracy requirements. Future research will seek to efficiently calculate JR in map-reduce proving that CS2 excel with approximate query processing in big data.

\section{Acknowledgement}

This research is partially supported by Cushwa Shearing Graduate Fellowship, University Research Council Grant, and Research Professorship Award at Youngstown State University, and Amazon Research and Education Grant.

\section{References}

[1] Swarup Acharya, Phillip B. Gibbons, Viswanath Poosala, and Sridhar Ramaswamy. Join synopses for approximate query answering. SIGMOD Rec., 28(2):275-286, June 1999.

[2] Dhruba Borthakur. The hadoop distributed file system: Architecture and design. Hadoop Project Website, 11(2007):21, 2007.

[3] Surajit Chaudhuri, Rajeev Motwani, and Vivek Narasayya. On random sampling over joins. In ACM SIGMOD Record, volume 28, pages 263-274. ACM, 1999.

[4] Transaction Processing Performance Council. Tpc-h benchmark specification. Published at http://www. tcp. org/hspec. html, 21:592-603, 2008.

[5] Giuseppe DeCandia, Deniz Hastorun, Madan Jampani, Gunavardhan Kakulapati, Avinash Lakshman, Alex Pilchin, Swaminathan Sivasubramanian, Peter Vosshall, and Werner Vogels. Dynamo: amazon's highly available key-value store. In ACM SIGOPS operating systems review, volume 41, pages 205-220. ACM, 2007.

[6] Amir Gandomi and Murtaza Haider. Beyond the hype: Big data concepts, methods, and analytics. International Journal of Information Management, 35(2):137-144, 2015.

[7] Wen-Chi Hou, Gultekin Ozsoyoglu, and Baldeo K Taneja. Statistical estimators for relational algebra expressions. In Proc. SIGMOD'88, pages 276-287. ACM, 1988.

[8] Yannis E Ioannidis. Query optimization. ACM Computing Surveys (CSUR), 28(1):121-123, 1996.

[9] Bernard Marr. How much data do we create every day? the mind-blowing stats everyone should read, Jul 2018.

[10] Frank Olken. Random sampling from databases. PhD thesis, University of California, Berkeley, 1993.

[11] Arun Swami. Optimization of large join queries: combining heuristics and combinatorial techniques. In ACM SIGMOD Record, volume 18, pages 367-376. ACM, 1989.

[12] Ashish Thusoo, Joydeep Sen Sarma, Namit Jain, Zheng Shao, Prasad Chakka, Suresh Anthony, Hao Liu, Pete Wyckoff, and Raghotham Murthy. Hive: a warehousing solution over a map-reduce framework. Proceedings of the VLDB Endowment, 2(2):1626-1629, 2009.

[13] Mark Van Rijmenam. A short history of big data, 2015.

[14] Mehul Nalin Vora. Hadoop-HBase for large-scale data. In Proceedings of 2011 International Conference on Computer Science and Network Technology, volume 1, pages 601-605. IEEE, 2011.

[15] Tom White. Hadoop: The definitive guide. O'Reilly Media, Inc, 2012.

[16] Feng Yu, Wen-Chi Hou, Cheng Luo, Dunren Che, and Mengxia Zhu. CS2: A New Database Synopsis for Query Estimation. In Proc. SIGMOD'13, pages 469-480, New York, NY, USA, 2013. ACM. 